\title{
Identification of Primary Shape Descriptors on 3D Scanned Particles
}

\author{
Balázs Ludmány ${ }^{1 *}$, Gábor Domokos²,3 \\ ${ }^{1}$ Department of Control Engineering and Information Technology, Faculty of Electrical Engineering and Informatics, \\ Budapest University of Technology and Economics, H-1521 Budapest, P.O.B. 91, Hungary \\ 2 MTA-BME Morphodynamics Research Group, H-1521 Budapest, P.O.B. 91, Hungary \\ ${ }^{3}$ Department of Mechanics, Materials and Structures, Faculty of Architecture, Budapest University of Technology and Economics, \\ H-1521 Budapest, P.O.B. 91, Hungary \\ *Corresponding author, email: ludmany.balazs@cloud.bme.hu
}

Received: 29 March 2018, Accepted: 25 April 2018, Published online: 08 May 2018

\begin{abstract}
The number of global mechanical equilibria as a shape descriptor (among others, for sedimentary particles) is at the forefront of current geophysical research. Although the technology is already available to provide scanned, 3D images of the particles (appearing as fine spatial discretization of smooth surfaces), nevertheless, the automated identification and measurement of global equilibria on such 3D images has not been solved so far. The main difficulty lies in the algorithmic distinction between local equilibria (associated with the small un-evenness of the pebble's surface) and global equilibria, associated with the overall shape. The former are easily measured, however, only the latter provide meaningful physical information. Here we provide and illustrate an algorithm to detect global equilibrium points on a finely discretized, polyhedral surface provided by 3D scan of sedimentary particles.
\end{abstract}

\section{Keywords}

finely discretized surface, convex polyhedron, level set, static equilibrium, 3D scan, sedimentary particle

\section{Introduction}

The shape evolution of particles of various sizes has been increasingly in the focus of geophysical and planetary research for the past decades [1-7]. The main goal of this research area is to extract information on the provenance of particles based alone on their current shape. This type of information can be crucial: analysis of the shape of Martian pebbles led to the confirmation of intense fluvial activity on ancient Mars [6] and the analysis of the shape of the asteroid 'Oumuamua led to confirm its interstellar origin [7]. The decoding of shape evolution relies on mathematical models, most often operating with nonlinear geometric partial differential equations (PDEs) $[1,8,9]$ and on laboratory and field measurements, which, depending on the subject, may range from analysis of photos, photometric imaging, hand-held manual measurements to 3D scanning. To bridge the gap between mathematical theory and the physical process, specific shape descriptors are used. Such shape descriptors are carefully selected on three, rather different and often contradicting type of criteria:
1. one seeks shape descriptors on the evolution of which rigorous mathematical results are either available or in sight.

2. one seeks shape descriptors on the evolution of which the available mathematical results make robust predictions in the sense that qualitative features of the evolution are not sensitive to model parameters or to unavoidable measurement errors.

3. one seeks shape descriptors on which experimental data has been already accumulated so current results can be compared to earlier ones.

For many shape descriptors, these criteria do not overlap. In some cases there is partial overlap; these cases we list below:

a) axis ratios (for main approximate axes $a>b>c$ we have $p=c / a, q=b / a$ ) have been broadly used in the geological literature [10-12], however, few rigorous mathematical results are available [13].

b) the isoperimetric ratio $I$ is related to the classical 
roundness measure of pebbles [14], it is increasingly used in recent works $[5,6,15]$ and there are also mathematical results available $[16,17]$.

c) the number $N$ of mechanical equilibria (i.e. the number $N$ of critical points of the distance function measured from the center of mass) has also been used increasingly in recent years $[5,15,18,19]$ and there is significant mathematical background available [17, 20, 21].

Although $N$ (in particular, combined with $I$ ) is a very powerful and promising shape descriptor, the digital measurement of mechanical equilibria poses considerable challenges.

Most notably, as it has been shown [22, 23] these equilibria appear on two separate scales. Roughly speaking, the global value $N$ corresponds to the approximation of the particle's convex hull by a sufficiently smooth surface while the local value $N^{\Delta}$ corresponds to a polyhedral approximation (with faces of maximal diameter $\Delta$ ) of the above-mentioned surface. After 3D scanning an object, we have discrete points with no higher level of information. Triangulation of the point set can be accomplished, resulting in a multi-faceted polyhedron on which the value of $N^{\Delta}$ can be readily obtained. However, in general, $\lim _{\Delta \rightarrow 0} N^{\Delta}>N$. As it has been pointed out in [22], local equilibria appear in flocks which are spatially localized around the locations of global equilibria. This poses a considerable problem for measurements: while only $N^{\Delta}$ is directly available from any 3D scanned dataset, the physically relevant quantity is $N$.

To obtain $N$ based on $N^{\Delta}$, one needs some artificial "blurring" of the data. One obvious choice would be a smoothing algorithm. Here we take an alternative route and perform the blurring directly on the polyhedron. This approach offers the advantage that visual comparison between the original and the blurred dataset is possible on one and the same surface. The core of the algorithm is to construct the level sets of the distance function measured from the center of mass (also known from cartography as contour lines) of the polyhedron and apply a simple sizebased filter for these level sets. As we will illustrate in Section 3, this proves to be a robust and reliable tool.

Our approach has yet another advantage. All previously described shape descriptors associate the investigated shape with one or two scalars and these descriptors are commonly called primary descriptors. However, there exist deeper level (secondary, tertiary etc.) classification schemes where the investigated shape is associated with more complex objects, e.g. graphs. One of the secondary schemes associates the shape with a so-called Reeb graph [24] and, as we will point out later, our algorithm automatically provides the Reeb graph as an output. However, the current paper is about primary shape descriptors and here we will not explore the deeper, secondary information offered by our algorithm.

It might be of interest to note that a human observer performs a "blurring" very similar to the one performed by our algorithm. However, the computerized method has the advantage that the amount of blurring is easily quantified and controlled. Throughout the paper we assume a convex, triangulated surface as input which can be readily obtained as the convex hull of a 3D scanned particle.

\section{The algorithm}

In this description we use the original cartographic terminology but with slightly modified semantics. In our case contour lines are curves on a body's surface along which the distance from a pre-defined point is equivalent. Usually the body's center of mass is used but the algorithm is not limited to that point. There might be several independent contour lines at a given distance, think of one around each vertex of a cube for example. When we draw the contour lines at several different distances we can get a rough estimation of the surface. This is equivalent to intersecting the surface with spheres of increasing radii but the same center. We generate the list of these spheres in advance and use indices to refer to them later in the algorithm.

When doing so, a contour line divides the surface to two separate parts, with neighboring contour lines on both sides. There are cases when we intuitively describe it as one contour line containing the other. For example as we get closer to the vertex of a cube, we see progressively smaller contour lines which are seemingly in one another. Something similar happens on the faces of the cube, but in that case contour lines "contain" the lower level ones unlike in the previous case.

To solve this confusion we only describe the adjacency of contour lines instead of a containing relationship. We build a graph with edges representing the contour lines and vertices representing the regions between them. The edges can then be directed upwards from lower levels or the other way around. These two cases translate to the lower levels containing the higher ones and the opposite, which have to be handled separately. The area of the regions between the two contour lines is stored in the vertices as well to make it easier for algorithms using the graph to track their progress. 
The biggest strength of the algorithm is that it does not use geometric primitives for comparison. Every point and section is described in terms of vertex and edge identifiers of the input surface or some similar integer index. This allows us to avoid floating point arithmetic and all of its pitfalls.

\subsection{Processing a face}

A contour line is either a complete circle on a single face of the surface or is made up of several connecting circular arcs on adjacent faces. A circular arc is defined by a supporting circle and two endpoints on that. We handle the two cases together by making the definition of endpoints optional.

We can get the supporting circle by calculating the intersection of the face's plane and a sphere with the radius of the distance at which we want to draw the contour line. We do the same with the edges of the face and the sphere to get the endpoints. These points divide the circle to two or more arcs, some of which lay inside the triangle while the others are outside. To determine this we walk around the face's edges and vertices in a counterclockwise fashion as seen on Fig. 1. If during this we get to a vertex that is outside of the sphere then the previous and next intersections on the edges are endpoints of an arc inside the triangle.

Besides the supporting circle and the endpoints we also store which vertices we visited between the endpoints in this step as well as the index of the edges the endpoints lay on.

It is also possible that none of the shapes intersect, in which instance we simply move on to looking for the next contour line on the face. In another case the sphere might intersect the face's plane but not the edges, which means that either the face contains the complete circle of intersection, or the circle contains the face, or they are disjoint. The first case is handled by storing the circle as a contour line on its own. The second one means that there is no more contour lines on this face. The last one is the same as if there was no intersection at all.

Similarly to the contour lines, the regions between them is also made up of smaller pieces. These subregions are bound by edges of the face and arcs of consecutive contour lines. At this point we do not know if contour lines of the same distance connect somewhere outside the face therefore we treat them as if they were separate. We use the vertices bundled with the arcs in the previous step to match arcs on a given level to the ones on the next. If there is a mutual vertex then those two arcs belong to the same subregion. For example on Fig. 2 the contour lines on the right border the same subregion because we would bundle $\{B, C\}$ to the blue one while $\{\mathrm{B}\}$ and $\{\mathrm{C}\}$ to the two green ones.

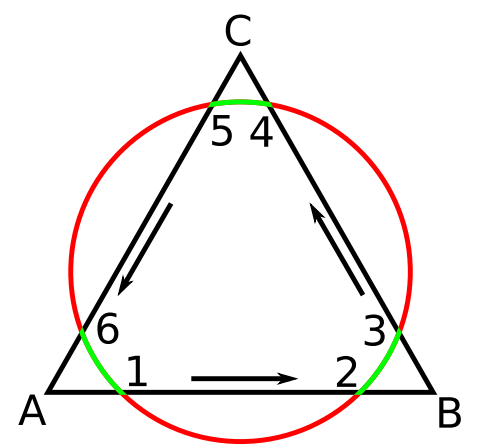

Fig. 1 Arc segments of a single contour line appearing on the face $\mathrm{ABC}$. Contour line associated with the plane carrying $\mathrm{ABC}$ is a circle. Intersections of the circle with edges of $\mathrm{ABC}$ are numbered consecutively. Subsequently arcs inside the face are identified (green arcs).

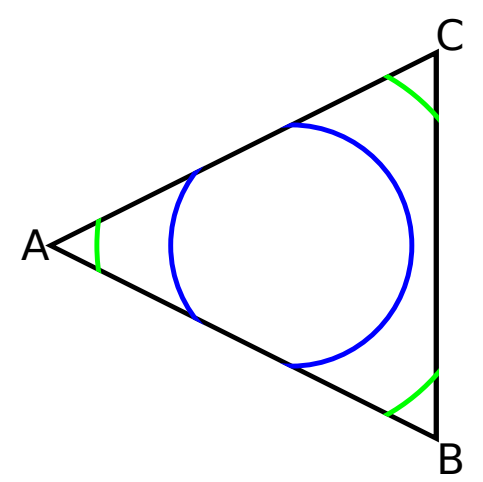

Fig. 2 Two consecutive contour lines on a face

On the level adjacency graph vertices represent regions while edges represent contour lines bordering them. We do not create this graph right away but rather one representing the subregions and their connections first. Two subregions are adjacent either through an arc on a face or across a common edge between adjacent faces.

The first case is the simpler one. When we iterate over the arcs of a certain level we also insert a vertex into the graph for each of them. We connect these to the vertices of arcs they form a contiguous subregion with. For every face we add a default vertex as well which we connect to when no previous sphere has intersected the triangle yet. On Fig. 3 there is an example where the color of a vertex in the graph corresponds to the color of the arc which was the reason for adding it.

We also store the area of the subregions in the vertices. This is the only place where we use the floating point coordinates of intersection points. First we calculate the area of the polygon defined by the endpoints of bounding arcs and edges. Then we add the area of arc segments of higher levels and subtract the lower one's. Later when we collect the subregions making up a region we sum these values too. 


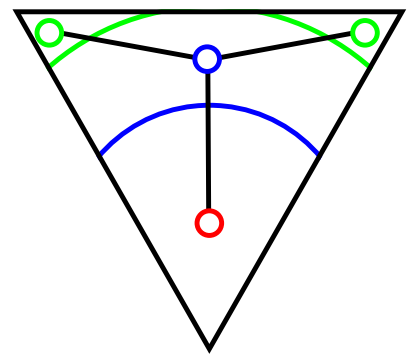

Fig. 3 Graph of adjacent subregions on a face where arcs and vertices belonging to the same level have the same color

Finding the continuity across two faces is more complicated because both of them has to be processed first. In case only one of them has been visited yet, we store the vertex of the graph that belongs to the subregion and a description of the common edge's section. This section will border a different subregion on the adjacent face, and when we find it we can connect the vertices we associated with them. Fig. 4 shows two such subregions with the common section highlighted.

If an edge is completely between two levels then its identifier is enough information to match up the incident subregions. Otherwise a contour line is intersecting the edge, in which case we have to describe the part of the edge between this and the next intersection. As a first approach we might want to store not only the edge's index but the index of the sphere we used to find the contour line. But these two shapes can meet in two distinct points which might belong to two separate contour lines. The way to tell them apart is to store whether they are the first (source/S) or last (target/T) point of an arc when going around faces. There is going to be only one of each because of the counterclockwise iteration. When visiting the same edge from the opposite side they are going to be switched up, but we can match up source to target and vice versa. Fig. 5 shows how this technique can be used to add the dotted edges between the vertices of the already existing subgraphs.

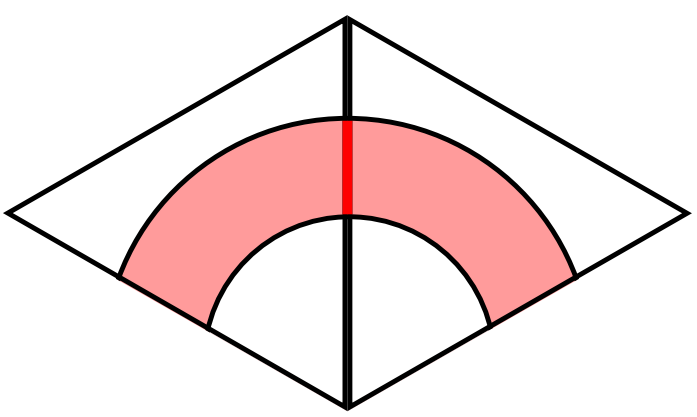

Fig. 4 Common section (red) between subregions (pink) on adjacent faces

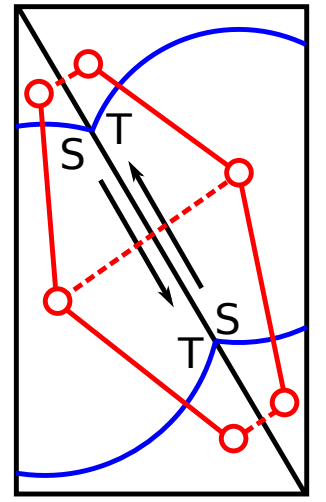

Fig. 5 Graph of adjacent subregions between two faces with dashed lines representing edges of the graph over face boundaries

\subsection{Level adjacency graph}

We run the previous steps on all the faces of the surface and we get a graph that describes all the subregions of the surface. The next step is merging the vertices that are between the same contour lines. We do this by getting the subgraph that has only the vertices corresponding to subregions between two consecutive levels. Merging every connected component in the subgraph into a single vertex and repeating it for every pair of levels creates the level adjacency graph. Fig. 6 gives an example of how the merging affects the graph. The Reeb graph [24] associated with the surface is easily retrieved based on this adjacency graph.

Edges of the level adjacency graph can be directed from lower levels to higher ones. A leaf with no in-edges in such a graph corresponds to an area containing a local minimum of the surface. By starting off from such a leaf and following out-edges we get to concentric contour lines of higher and higher levels. Doing so with the edges flipped is a walk down from the maximums. At every step we calculate the area bordered by the latest contour line and relate it to the surface area of the complete mesh. The final goal of the algorithm is to identify and count the contour lines this relative area of which is just higher than a pre-set value. The intuitive description of this parameter is that it sets how "blind" the algorithm should be to minor details. In Section 3 we show

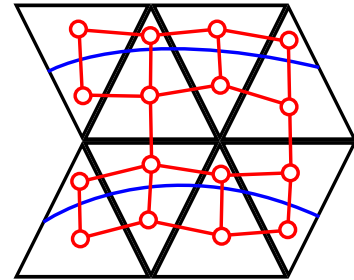

before merging

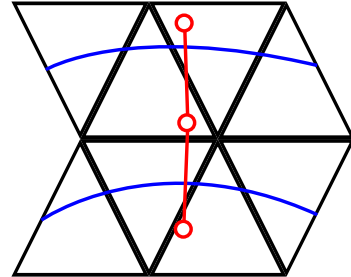

after merging
Fig. 6 Level adjacency graph 
this "blurring" in action. By traversing the original graph we get the number of stable equilibria while repeating it with the edges flipped yields the number of unstable ones.

\section{Results}

In this section, we illustrate the algorithm on some examples. In the first example, we show how our algorithm performs the "blurring" required to obtain global equilibria based on local information. Fig. 7 illustrates the vicinity of an equilibrium point of the smooth surface where the discretization results in a flock containing 4 distinct equilibria. By omitting level sets below a critical (pre-set) area, the flock is merged and appears as a single equilibrium point reflecting the geometry of the underlying smooth surface.

In the second example, we illustrate that the global equilibria identified by our algorithm can be easily realized in a hand experiment. Fig. 8 shows the global stable positions of a pebble both in the computational model and in an experiment. The pictures on the left show the contour lines with the stable ones highlighted in green. These were then marked on the real pebble for reference. The pebble was then placed on a glass table and photographed from underneath proving that it is in fact stable in all three positions the program showed.

\section{Summary}

In the article we developed an algorithm suitable to identify primary shape descriptors of a 3D scanned particle. Some of these are trivially computed; however, one of the most significant shape descriptors, the number of global equilibrium points, is hard to obtain because of the unavoidable discretization. We achieved this goal by constructing equidistant level sets and filtering them by minimal size. We also presented an algorithm that can construct the level adjacency graph with the help of contour lines. Finally, we gave a brief example of how the data structure can be utilized.

Based on the calculated values it is possible to obtain the primary equilibrium classification for any particle in an automated manner. This capability will help to understand the natural abrasion of pebbles and how their shape and number of equilibrium points change over their lifetime.
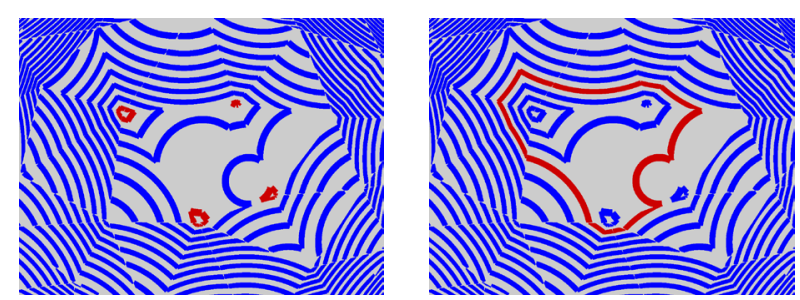

Fig. 7 Merging 4 local equilibria. Left panel: blue (regular) contour lines and 4 red contour lines marking local equilibria. Right panel: blue (regular) contour lines and one red contour line marking one single global equilibrium
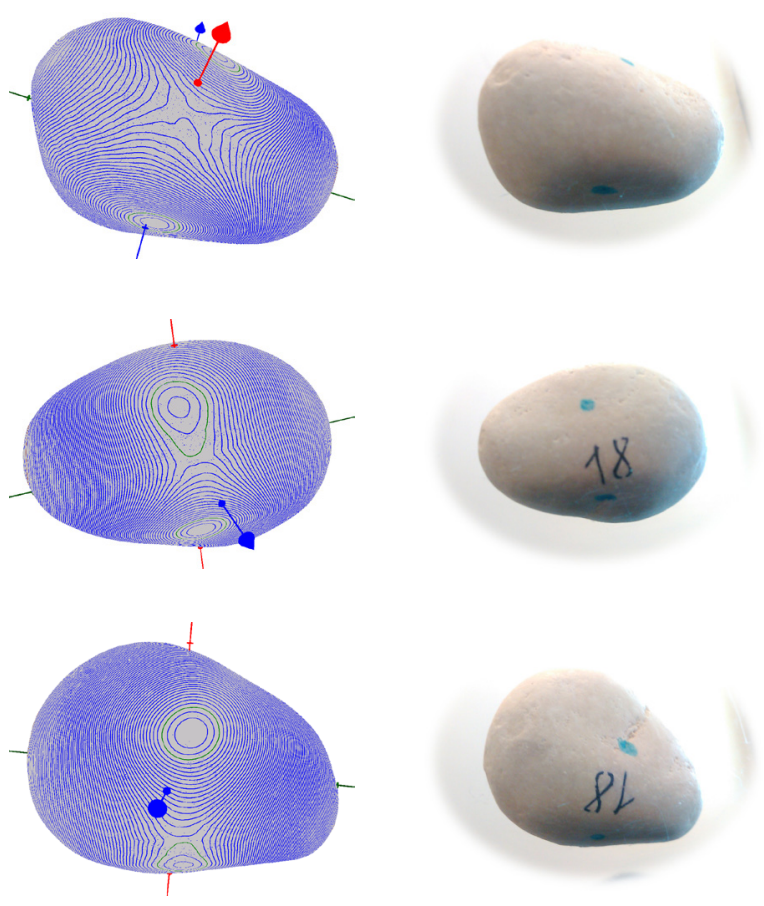

Fig. 8 Experimental verification of global equilibria. Right panel: pebble exhibiting 3 global stable equilibria, marked with dots on the surface of the pebble. Three different views are displayed below each other. Left panel: 3D scanned image of the pebble. Regular contour lines appear in blue color while global equilibria are surrounded by green contour line. Same three views displayed as in right panel.

\section{Acknowledgement}

This work has been supported by NKFIH research grant 119245. The authors thank András Sipos and Imre Szeberényi for valuable advice on multiple topics and also thank an anonymous referee for making very useful suggestions and comments. 


\section{References}

[1] Bloore, F. J. "The shape of pebbles", Journal of the International Association for Mathematical Geology, 9(2), pp. 113-122, 1977. https://doi.org/10.1007/BF02312507

[2] Attal, M., Lavé, J. "Changes of bedload characteristics along the Marsyandi River (central Nepal): Implications for understanding hillslope sediment supply, sediment load evolution along fluvial networks, and denudation in active orogenic belts", Geological Society of America Special Papers, 398, pp. 143-171, 2006. https://doi.org/10.1130/2006.2398(09)

[3] Durian, D. J., Bideaud, H., Duringer, P., Schröder, A., Thalmann, F., Marques, C. "What Is in a Pebble Shape?", Physical Review Letters, 97(2), 2006.

https://doi.org/10.1103/PhysRevLett.97.028001

[4] Domokos, G., Jerolmack, D. J., Sipos, A. Á., Török, Á. "How River Rocks Round: Resolving the Shape-Size Paradox", PLoS ONE, 9(2), p. e88657, 2014.

https://doi.org/10.1371/journal.pone.0088657

[5] Litwin Miller, K., Szabó, T., Jerolmack, D. J., Domokos, G. "Quantifying the significance of abrasion and selective transport for downstream fluvial grain size evolution", Journal of Geophysical Research: Earth Surface, p. 2014JF003156, 2014. https://doi.org/10.1002/2014JF003156

[6] Szabó, T., Domokos, G., Grotzinger, J. P., Jerolmack, D. J. "Reconstructing the transport history of pebbles on Mars", Nature Communications, 6, p. 8366, 2015.

https://doi.org/10.1038/ncomms9366

[7] Domokos, G., Sipos, A. Á., Várkonyi, P. L., Szabó, G. M. "Explaining the elongated shape of 'Oumuamua by the Eikonal abrasion model", Research Notes of the AAS, 1(1), 2017. https://doi.org/10.3847/2515-5172/aaa12f

[8] Firey, W. J. "Shapes of worn stones", Mathematika, 21(1), pp. 1-11, 1974. https://doi.org/10.1112/S0025579300005714

[9] Gage, M., Hamilton, R. S. "The heat equation shrinking convex plane curves", Journal of Differential Equations, 23, pp. 69-96, 1986.

https://doi.org/10.4310/jdg/1214439902

[10] Blott, S. J., Pye, K. "Particle shape: A review and new methods of characterization and classification", Sedimentology, 55, pp. 31-63, 2008. https://doi.org/10.1111/j.1365-3091.2007.00892.x

[11] Landon, R. E. "An Analysis of Beach Pebble Abrasion and Transportation", The Journal of Geology, 38(5), pp. 437-446, 1930. https://doi.org/10.1086/623739

[12] Szabó, T., Fityus, S., Domokos, G. "Abrasion model of downstream changes in grain shape and size along the Williams River, Australia", Journal of Geophysical Research: Earth Surface, 118(4), pp. 2059-2071, 2013.

https://doi.org/10.1002/jgrf.20142
[13] Domokos, G., Gibbons, G. W. "The evolution of pebble size and shape in space and time", Proceddings of the Royasl Society London, Series A, 468(2146), pp. 3059-3079, 2012. https://doi.org/10.1098/rspa.2011.0562

[14] Krumbein, W. C. "The effects of abrasion on the size, shape and roundness of rock fragments", The Journal of Geology, 49(5), pp. 482-520, 1941. https://doi.org/10.1086/624985

[15] Novák-Szabó, T., Sipos, A. Á., Shaw, S., Bertoni, D., Pozzebon, A., Grottoli, E., Sarti, G., Ciavola, P., Domokos, G., Jerolmack, D. J. "Universal characteristics of particle shape evolution by bed-load chipping", Science Advances, 4(3), 2018. https://doi.org/10.1126/sciadv.aao4946

[16] Gage, M. E. "An isoperimetric inequality with applications to curve shortening", Duke Mathematical Journal, 50(4), pp. 12251229, 1983. https://doi.org/10.1215/S0012-7094-83-05052-4

[17] Domokos, G., Lángi, Z. "The isoperimetric quotient of a convex body decreases monotonically under the Eikonal abrasion model", 2018. [online] Available at: http://arxiv.org/abs/1801.06796 [Accessed: 21 April 2018]

[18] Domokos, G., Sipos, A., Szabó, T., Várkonyi, P. "Pebbles, shapes, and equilibria", Mathematical Geosciences, 42(1), pp. 29-47, 2010. https://doi.org/10.1007/s11004-009-9250-4

[19] Domokos, G., Kun, F., Sipos, A. Á., Szabó, T. "Universality of fragment shapes", Scientific Reports, 5, p. 9147, 2015. https://doi.org/10.1038/srep09147

[20] Grayson, M. "The heat equation shrinks embedded plane curves to round points", Journal of Differential Geometry, 26, pp. 285-374, 1987.

https://doi.org/10.4310/jdg/1214441371

[21] Domokos, G. "Monotonicity of Spatial Critical Points Evolving Under Curvature-Driven Flows", Journal of Nonlinear Science, 25(2), pp. 247-275, 2015. https://doi.org/10.1007/s00332-014-9228-3

[22] Domokos, G., Lángi, Z., Szabó, T. "On the equilibria of finely discretized curves and surfaces", Monatshefte für Mathematik, 168, pp. 321-345, 2011. https://doi.org/10.1007/s00605-011-0361-x

[23] Domokos, G., Sipos, A. Á., Szabó, T. "The mechanics of rocking stones: equilibria on separated scales", Mathematical Geosciences, 44, pp. 71-89, 2012. https://doi.org/10.1007/s11004-011-9378-x

[24] Nicolaescu, L. I. "Counting Morse functions on the 2-sphere", Compositio Mathematica, 144(5), pp. 1081-1106, 2008. https://doi.org/10.1112/S0010437X08003680 